Revue

de Sémantique

et Pragmatique

\section{Revue de Sémantique et Pragmatique}

40 | 2016

Exclamation et intersubjectivité

\title{
Exclamation et intersubjectivité en allemand
}

\section{Anne Larrory-Wunder}

\section{(2) OpenEdition}

Journals

Édition électronique

URL : http://journals.openedition.org/rsp/313

DOI : $10.4000 /$ rsp.313

ISSN : 2610-4377

\section{Éditeur}

Presses universitaires d'Orléans

\section{Édition imprimée}

Date de publication : 1 mars 2017

Pagination : 79-98

ISSN : 1285-4093

\section{Référence électronique}

Anne Larrory-Wunder, "Exclamation et intersubjectivité en allemand », Revue de Sémantique et

Pragmatique [En ligne], 40 | 2016, mis en ligne le 01 mars 2018, consulté le 30 avril 2019. URL : http:// journals.openedition.org/rsp/313 ; DOI : 10.4000/rsp.313 


\title{
EXCLAMATION ET INTERSUBJECTIVITÉ EN ALLEMAND
}

\author{
Anne Larrory-Wunder \\ CEREG EA 4223, Université Sorbonne Nouvelle - Paris 3
}

Dans cette contribution, nous examinerons, à partir de données de l'allemand - essentiellement un corpus écrit ${ }^{1}$ - des éléments plaidant en faveur d'une valeur intersubjective de l'énoncé exclamatif, déjà défendue par d'autres auteurs (notamment Gaubert 2001 pour l'allemand, Kerfelec 2009, Guillaume / Baumer 2014 pour l'anglais), mais que nous appuierons ici sur l'analyse de certaines configurations de marqueurs en allemand, en mobilisant des outils inspirés par les travaux de Culioli (2002). Posant la fonction commentative comme définitoire de l'énoncé exclamatif, nous montrerons que l'énoncé exclamatif en écho constitue un révélateur tout autant de cette fonction que de la valeur intersubjective de ce commentaire : dans ce contexte particulier, l'énoncé exclamatif se démarque à la fois de l'interrogation et de l'assertion et met en évidence un hiatus entre «l'état de croyance » du locuteur et celui de l'interlocuteur. Nous montrerons ensuite trois types d'exclamatives où se manifeste particulièrement la dimension intersubjective, par la sollicitation d'un co-énonciateur, le ménagement de l'interlocuteur, le renvoi à une instance énonciative imaginaire. Nous nous pencherons enfin sur quelques indices de la proximité à la fois contextuelle et fonctionnelle entre les exclamatives et certaines injonctives.

${ }^{1}$ Le corpus de référence que nous avons rassemblé (et exploité dans Larrory 2002) est constitué surtout de textes littéraires fictionnels (romans et nouvelles, théâtre, pièces radiophoniques, littérature de jeunesse), dans une moindre mesure, de textes de presse, d'extraits de bandes dessinées, de films de fiction et d'exemples oraux relevés par nous. Dans le présent article, nous complétons ponctuellement ces données par le recours au Deutsches Referenzkorpus (DeReKo) ou à des requêtes aléatoires sur Internet. 


\section{LA THÈSE DE L'ÉNONCÉ MONOLOGIQUE ET LA FONCTION COMMENTATIVE}

L'énoncé exclamatif est parfois décrit comme un énoncé qui ne serait pas véritablement adressé. Pour l'allemand, Valentin écrit ainsi :

«Sur la base d'une certaine parenté formelle, on a souvent rapproché les exclamations des interrogatives (....) Mais une exclamation ne sert de toute évidence pas à faire réagir un interlocuteur, ce qui est confirmé par son intonation de type descendant après un sommet situé en général près du début. » (Valentin 1989, p. 244) et encore :

« les exclamations ne paraissent jamais présupposer vraiment la présence d'un allocuté, qui, de toute façon, ne serait pas là pour contredire : l'interlocuteur, s'il y en a un, ne peut ni accuser son partenaire de mensonge, ni chercher à nuancer son propos ; il ne peut que refuser en bloc et son vis-à-vis et ce que celui-ci a dit : $d u$ sagst es! [C'est toi qui le dis! ALW]» (loc. cit.)

De ce point de vue se pose la question de savoir quelle intention communicative portent les énoncés exclamatifs :

« Nous pensons pour notre part que cette intention n'est pas de communication, si communication implique partenaire. Certes, du bist mir einer! [Tu es quelqu'un, toi ! ALW] contient un $d u[t u]$, mais ce n'est plus qu'un spectateur, c'est à peine un auditeur, et ce $d u$ ne pourra jamais rien répliquer. » (ibid., p. 247)

À l'appui de la thèse selon laquelle l'énoncé exclamatif serait essentiellement monologique, on peut arguer que le monologue intérieur est un de ses contextes d'emplois privilégiés dans les récits littéraires; l'énoncé exclamatif en constitue même un des marqueurs d'ouverture, un indice de la présence d'un discours indirect libre :

(1) Er befreite sich aus der Menschensammlung [a] und ging ihr hinterher, im Abstand von ungefähr zwanzig Metern. [b] Wie langsam seine Mutter lief! [c] Es erschien ihm plötzlich erschreckend, wie sie gealtert war, in kürzester Zeit, [d] sie mußte einen förmlichen Alterungsschub gehabt haben; [e] sie ging unsicher, übervorsichtig, gebeugt unter der Last der Taschen, ihr Blick war zu Boden gerichtet, wahrscheinlich wegen des grünen Stars, unter dem ihre Augen litten. (Hilbig, Provisorium, p. 167)

[Il se dégagea des gens agglutinés et la suivit, à un intervalle d'une vingtaine de mètres. Comme sa mère avançait lentement ! Il trouvait soudain effrayant combien elle avait vieilli, en si peu de temps, c'était certainement arrivé d'un seul coup ; elle marchait sans assurance, avec une extrême prudence, courbée sous le fardeau de ses sacs, le regard rivé au sol, sans doute à cause du glaucome dont ses yeux étaient atteints. (trad. Vergne-Cain et Rudent, p. 138)]

Dans ce passage narratif, l'énoncé exclamatif [b] marque une rupture. Il ne renvoie pas à un événement du récit, mais constitue un commentaire, que le lecteur attribue au personnage principal, placé en position de percevoir certains faits [a], et il ouvre sur sa droite un segment de texte $[\mathrm{c}, \mathrm{d}, \mathrm{e}]$ où s'exprime le 
point de vue ${ }^{2}$ de ce personnage, où sont détaillés des aspects de sa perception initiale et des pensées associées à cette perception (cf. notamment l'emploi du verbe de point de vue erschien [trouvait] et du verbe de modalisation mußte [certainement]).

Cette dimension commentative constitue un caractère essentiel de l'exclamation. Les limites de cet article ne permettent pas d'entrer dans le détail des débats controversés autour de la définition de l'exclamation ${ }^{3}$; selon nous, l'énoncé exclamatif peut être défini comme un type d'énoncé (au même titre que l'énoncé déclaratif, interrogatif ou injonctif) associant une certaine configuration de marquages syntaxiques, morphologiques, lexicaux, prosodiques et une fonction, celle d'exprimer un commentaire affectif sur un état de choses donné (Larrory 2002, 2004). Attribuer à l'énoncé exclamatif la valeur d'un commentaire implique certainement qu'on ne puisse envisager qu'il réalise un acte illocutoire à proprement parler, au sens de Ducrot (1984), qui considère que les actes illocutoires entraînent une modification quasi juridique de la relation entre les partenaires d'une interaction ${ }^{4}$. Une telle définition ne laisse pas non plus nécessairement place à l'interlocuteur. Le commentaire exclamatif est-il d'une quelconque façon adressé ? De quelle manière l'interlocuteur est-il impliqué dans cette énonciation? Les cas analysés ci-après, qui se situent dans des contextes de monologue comme de dialogue, montrent que l'énonciation exclamative met en jeu, de diverses manières selon les formes concernées, une autre position subjective que celle de l'énonciateur, à laquelle peut être identifié l'interlocuteur ou un co-énonciateur imaginaires.

2 au sens de Rabatel (1998).

${ }^{3}$ Dans les travaux sur l'allemand, ces débats portent essentiellement sur la question du statut de l'exclamation. D'Avis (2013) résume ces débats en distinguant deux approches de la recherche (Ansätze), une approche consistant à « dériver » l'exclamation d'autres types de phrases (Ableitungsansatz) et une approche consistant à « regrouper » les phrases ayant une «fonction exclamative » (Zuordnungsansatz) : dans la première perspective, les types de phrases sont définis comme des types syntaxiques et l'exclamation, qui ne repose pas sur un type syntaxique propre, se rattache au type assertif ou au type interrogatif (cf. notamment Fries 1988, Rosengren 1992, d'Avis 2001) ; dans la seconde perspective, l'exclamation représente une «modalité phrastique », définie par des critères formels et fonctionnels (cf. notamment Näf 1987, Altmann 1993). C'est à ce deuxième type d'approche que l'on peut rattacher notre proposition de définition.

${ }^{4}$ « Dire qu'un énoncé possède, selon les termes de la philosophie du langage, une force illocutoire, c'est pour moi dire qu'il attribue à son énonciation un pouvoir « juridique », celui d'obliger à agir (dans le cas d'une promesse ou d'un ordre), celui d'obliger à parler (dans le cas d'une interrogation), celui de rendre licite ce qui ne l'était pas (dans le cas d'une permission), etc. » (p. 183)

${ }^{5}$ au sens de Culioli (2002) : « (...) l'énonciateur en fait est une origine subjective qui se construit nécessairement comme intersubjective, c'est-à-dire que nous construisons toujours un co-énonciateur qui n'est pas forcément en chair et en os. Le locuteur, lui, c'est une personne physique, l'interlocuteur aussi » (p. 27-28). 


\section{L'ÉNONCÉ EXCLAMATIF EN ÉCHO}

Le contexte particulier de la reprise des propos d'autrui dans l'interlocution constitue un révélateur intéressant de la valeur exclamative ; il manifeste aussi une dimension intersubjective de l'énoncé exclamatif :

(2) C. zögerte mit der Antwort: Schreiben... geschrieben habe ich, Schriftsteller bin ich, wenn das etwas sagt.

Schriftsteller, wiederholte er, Schriftsteller! Gehts etwa darum, über die Verhältnisse hier etwas zu schreiben? Ich glaube, wir sind nicht gerade der letzte Schrei in den Zeitungen. (Hilbig, Provisorium p. 52-53)

[C. hésita sur la réponse. Ecrire... j'ai écrit, je suis écrivain, si ça veut dire quelque chose.

Ecrivain, répéta-t-il, écrivain ! Faut-il écrire sur ce qui se passe ici ? Nous ne sommes pas très cotés dans les journaux à mon avis. (trad. Vergne-Cain et Rudent, p. 45)]

(3) [Sur le bateau qui l'amène à New York, Karl a perdu son parapluie et sa valise et rencontre un homme qui semble pouvoir l'aider.] „Kennen Sie sich auf dem Schiff aus?" fragte Karl mißtrauisch, und es schien ihm, als hätte der sonst überzeugende Gedanke, daß auf dem leeren Schiff seine Sachen am besten zu finden sein würden, einen verborgenen Haken. „Ich bin doch Schiffsheizer“, sagte der Mann. „Sie sind Schiffsheizer!“ rief Karl freudig, als überstiege das alle Erwartungen, und sah, den Ellbogen aufgestützt, den Mann näher an. „Gerade vor der Kammer, wo ich mit dem Slowaken geschlafen habe, war eine Luke angebracht durch die man in den Maschinenraum sehen konnte." (Kafka, Der Verschollene, 12)

[- Vous connaissez bien le bateau ? demanda Karl avec méfiance.

Il avait l'impression que quelque chose clochait, dans cette idée par ailleurs convaincante que c'était sur le bateau désert que ses affaires se retrouveraient le mieux.

- Voyons, je suis soutier, dit l'homme.

- Vous êtes soutier, s'exclama joyeusement Karl comme s'il n'en espérait pas tant, en s'appuyant sur son coude pour examiner l'homme de plus près. Juste en face de la cabine que j'occupais avec le Slovaque, il y avait un hublot qui donnait dans la salle des machines. (trad. Lortholary, p. 21)]

Dans ces deux extraits, on a un énoncé de reprise, en écho, d'un propos originel ${ }^{6}$, marqué d'un point d'exclamation. Le contexte de reprise des propos d'autrui dans le dialogue permet d'observer que la reprise peut s'effectuer sur différents « modes », mais jamais sur un mode simplement assertif : on peut avoir une reprise « neutre » pour vérifier, «s'approprier» le contenu (Ecrivain, répéta-t-il); on pourrait imaginer une reprise sur un mode interrogatif, pour mettre en doute (ce serait : Ecrivain ?). Le mode exclamatif, dans les extraits

\footnotetext{
${ }^{6}$ moyennant transposition au système de repérage du nouveau locuteur.
} 
(2) et (3), sert à opposer les propos d'autrui à une attente (ce que le texte narratif souligne explicitement d'ailleurs : als überstiege das alle Erwartungen [comme s'il n'en espérait pas tant]; emploi de la particule énonciative etwa dans gehts etwa darum, présentant le contenu de l'énoncé comme une éventualité suggérée par le contexte mais dont le locuteur n'attend pas la réalisation : s'agit-il par hasard d'écrire sur ce qui se passe ici'): le locuteur signale alors le hiatus entre la représentation soulevée par les propos de l'interlocuteur et ses propres représentations. Pour Martin (1987), dans le cas de la phrase exclamative, il y a « une tension contradictoire » entre, d'une part, la proposition $p$, qui est assertée avec force et fait partie de l'univers actuel du locuteur et, d'autre part, une image d'univers où $p$ est possiblement faux. Ici, la reprise exclamative marque plutôt que l'univers de croyance du locuteur ne coïncide pas avec celui de l'interlocuteur qui asserte $p$.

La reprise exclamative porte parfois plus spécifiquement sur la forme de l'énoncé, par exemple sur l'emploi d'un terme comme en (4) :

(4) Er wand sich.

Ich sah ihm stumm zu.

„Rauchen Sie?“ fragte er und, als ich den Kopf schüttelte: „Darf ich Ihnen einen Kaffee... eine Erfrischung...? Vielleicht einen Cognac?“”

„Nein, vielen Dank!“ erwiderte ich. „Ich möchte nichts, Herr Professor! Nichts außer Ihrer Stellungnahme zum Fall Westphal!“

„Fall! Fall! Wie das klingt!“ rief er und mimte den Empörten. (Martin, Der Rest ist Sterben,H p. 56)

[Il se tortillait.

Je l'observais en silence.

- Vous fumez ? demanda-t-il et comme je secouais la tête, il ajouta :

- Puis-je vous offrir un café ? un rafraîchissement ? Peut-être un cognac ?

- Non, merci beaucoup ! répliquai-je, je ne veux rien, Monsieur le professeur ! Rien si ce n'est votre position quant au cas Westphal !

- Cas ! Cas ! Quel grand mot ! [litt. Comment ça sonne !] lança-t-il en feignant l'indignation. (trad. ALW)]

Dans cet exemple, la réduplication de Fall (maladroitement rendue dans la traduction en français) joue également un rôle. Elle s'apparente à ce que Watine (2015) a analysé en termes de "reprise-écho dissensuelle », dans des énoncés du type "La nature, la nature, c'est vite dit », où le segment rédupliqué la nature est une citation par l'énonciateur d'un propos d'un tiers) : comme elle l'écrit, « l'ensemble de la réduplication a pour fonction de souligner le dissensus interlocutif », avant que ce point de vue dissensuel, ici, ne s'exprime expli-

\footnotetext{
${ }^{7}$ Sur la valeur de etwa cf. Métrich / Faucher / Courdier 1999.
} 
citement par le biais de l'exclamative en wie, à valeur évaluative.

Un cas particulier de reprise est constitué par la forme exclamative en und (et) en allemand, que l'on trouve en (5) et (6) :

(5) EMMI Wissen Sie, was ich Ihnen sage? Kehren Sie vor Ihrer eigenen Tür lassen Sie mir die meine. Ich laß Ihnen ja auch die Ihre.

FRAU KARGES Wir haben uns auch nichts vorzuwerfen. Wir nicht.

EMMI Wenn Sie mich fragen: Sie sind neidisch, Frau Karges. Neidisch sind Sie und sonst nichts. Guten Tag. Sie geht.

FRAU KARGES Das ist wohl die Höhe. Ich und neidisch — wie meint sie denn das? (Fassbinder, Angst essen Seele auf)

[EMMI Vous voulez savoir ce que je vous dis ? Balayez devant votre porte laissez-moi la mienne. Je vous laisse bien la vôtre, moi.

MME KARGES Nous n'avons rien à nous reprocher, nous.

EMMI Si vous voulez mon avis : vous êtes jalouse, Mme Karges. Jalouse et rien de plus. Bonne journée. Elle s'en va.

MME KARGES Ça alors, c'est la meilleure. Moi, jalouse [litt : moi et jalouse]. Qu'est-ce qu'elle veut dire ? (trad. ALW)]

(6) „Ich bin organisiert“, sagt Pinneberg mürrisch. „Ich bin in'ner Gewerkschaft.“ „Emma! Mutter! Unser junger Mann ist in'ner Gewerkschaft? Wer hätte das gedacht! So schnieke und Gewerkschaft!“ (H. Fallada, Kleiner Mann, was nun?, p. 24)

[ « Je suis organisé », dit Pinneberg d'un ton bourru. « Je suis dans un syndicat» «Emma! Maman! Notre jeune homme est dans un syndicat? Qui l'eût cru? Quelqu'un d'aussi élégant, dans un syndicat ! [litt : aussi élégant et syndicat] (trad. ALW)]

La tournure en und a été analysée récemment par Bücker (2012) comme une « construction » au sens de la grammaire de construction appliquée à l'analyse conversationnelle. L'auteur lui attribue une fonction interactive : elle permet de renvoyer à un segment du propos de l'interlocuteur qui se trouve « recyclé » pour en faire un «objet de communication pertinent » de l'interaction (nouveau focus thématique); le locuteur initie une « réparation » évaluative, qui dans la plupart des cas conteste la validité ou la justification d'une affirmation qui précède ainsi que des positions discursives qu'elle propose.

Selon Bücker, cette construction est constituée d'un élément déictique, renvoyant presque toujours à un individu ou groupe d'individus, relié par und à un élément prédicatif ; il s'agit effectivement du schéma récurrent. Néanmoins, l'exemple (6) montre que l'expression référentielle déictique (der) peut ne pas être fournie et que la qualification s'appuie alors sur un référent sous-jacent.

Dans le contexte de reprise de propos d'autrui, la valeur intersubjective voire interactive de l'énoncé exclamatif, consistant en l'expression d'une posi- 
tion dissensuelle, semble donc se dégager assez clairement et n'autorise pas à maintenir une conception purement monologique de l'exclamation. Mais peuton étendre cette conclusion à d'autres contextes ?

\section{QUELLE VALEUR INTERSUBJECTIVE POUR LE COMMENTAIRE EXCLAMATIF ?}

Gaubert (2001), en repérant des marquages tels que Stellen Sie sich vor, stell dir vor (Imaginez-vous, imagine-toi), énoncés injonctifs que l'on trouve à proximité de certains énoncés exclamatifs, en mettant en avant ce qu'elle appelle des « perturbations » et « discordances », en vient à proposer que l'énoncé exclamatif est un appel pressant adressé au «co-énonciateur $»^{8}$ pour qu'il se représente un état de choses. L'incomplétude caractéristique de nombreuses structures exclamatives est interprétée comme l'une de ces «perturbations ». Un locuteur qui s'exclame, produit un énoncé tronqué, «ne fournit qu'une partie des informations nécessaires à la désignation complète des états de choses perçus » (p. 186) et, par là, sollicite du co-énonciateur un « effort de représentation ». Celui-ci « va participer à la construction du discours en effectuant un décodage très coopératif, qui ne se borne pas à être de la compréhension mais l'amène à se rapprocher de la perspective de l'énonciateur, à l'adopter, ne serait-ce que momentanément, afin d'être en mesure de saisir l'implicite, de co-construire le discours ». (p. 194)

Dans la suite de notre propos, nous voudrions aller dans le sens de la proposition de Gaubert en la précisant. Car tous les énoncés exclamatifs ne sollicitent pas de la même manière l'allocuté et l'on peut repérer, dans le foisonnement des tournures exclamatives, certains dispositifs énonciatifs qui exhibent plus particulièrement la dimension intersubjective et où se joue le positionnement de l'énonciateur par rapport à un interlocuteur fictif ou réel. Nous examinerons successivement les énoncés en was... alles, les énoncés en vielleicht et certains énoncés en wenn.

\subsection{MARQUAGES D'INTERSUBJECTIVITE DANS LES ENONCES EN WAS... ALLES}

On trouve en allemand le marqueur alles ('tout') en relation avec des exclamatives en w- : was (quoi/que), wer (qui) ou wo (où); alles est, dans cet emploi, invariable (neutre), ce qui a pu le faire ranger parmi les particules ${ }^{9}$. Il est presque toujours placé à distance de l'élément en $w$ -

Considérons l'exemple suivant :

(7) Sie beten! Onkel Jona nickte den beiden kurzen Wörtern mit höhnendem Einverständnis nach. Danach wurde er laut, sein Bauch begann, unter dem unordentlich geknöpften Hemd zu vibrieren. Ja, sie beten. Sie flehen. Sie flehen

\footnotetext{
${ }^{8}$ Le terme désigne chez l'auteur l'interlocuteur.

${ }^{9}$ Cf. Reis (1992).
} 
ihren Herrgott an, daß er sie siegen lasse und die Feinde verlieren. Was muten sie ihrem Gott alles zu. Ihre Kanonen soll er segnen, selbst in den Läufen der Pistolen soll sein guter Geist walten. (Härtling, Felix Guttmann, p. 24)

[Ils prient! Oncle Jona fit en écho à ces deux mots brefs, de la tête, un signe d'intelligence ironique. Puis sa voix s'éleva, son ventre se mit à vibrer sous la chemise boutonnée de travers. Oui, ils prient. Ils implorent, ils implorent leur bon Dieu pour qu'ils les fasse vaincre et fasse perdre leurs ennemis. Qu'est-ce qu'ils ne vont pas exiger de leur Dieu ! Il faut qu'il bénisse leur artillerie, et que sa bonne influence aille se fourrer jusque dans le canon de leurs pistolets. (trad. Porcell, p. 28)]

L'effet de alles est de suggérer que c'est un ensemble d'éléments qui vient instancier la place de l'objet syntaxique dans le procès 'sie ihrem Gott ( ) zumuten' [ils exigent ( ) de leur Dieu]. Le marqueur alles invite alors à rechercher ces éléments dans le co-texte : ici, on trouve l'énumération des prières attribuées par l'oncle Jona à ces 'ils' dont il commente l'action, d'autre part la présence d'une particule de focalisation selbst [même], qui établit pour cet ensemble d'activités une échelle valuée, sur laquelle la dernière activité décrite : in den Läufen der Pistolen soll sein guter Geist walten est située très haut ${ }^{10}$.

Mais l'implication de l'allocuté / récepteur se manifeste aussi dans trois marquages fréquemment associés à la structure was ... alles : la particule énonciative doch, le négateur nicht, la forme généralisante, le marqueur so.

Doch a en allemand un sens adversatif et peut aussi servir de connecteur textuel. Comme particule, il sert à construire, en l'insérant dans l'énoncé, une contre-position fictive pouvant être attribuée à un interlocuteur imaginaire, un « co-énonciateur $»^{11}$, pour la désamorcer par avance :

(8) Sammelleidenschaft

Heute, da unser Lebensunterhalt nicht mehr vom Sammeln abhängig ist ausser wir zählen das Anhäufen von «Fränkli» auch dazu - betreiben wir dieses noch als Hobby.

Was sich doch alles sammeln lässt. Von Briefmarken, Kaffeerahmdeckeli, Pins oder Parfümflacons bis zu Fingerhüten, Postcards oder Streichholzschächteli - alles findet einen Abnehmer. Die meisten konzentrieren sich beim Sammeln auf kostengünstige Gegenstände, die erst mit den Jahren wertvoll werden und dadurch vielleicht einmal einen grossen «Happen» Geld einbringen könnten. (DeReKo, St. Galler Tagblatt, 11.02.1999)

[La passion de collectionner

Aujourd'hui que notre subsistance ne dépend plus de la cueillette ${ }^{12}$ - sauf si nous y incluons l'accumulation des francs suisses -, nous en faisons encore un hobby.

\footnotetext{
${ }^{10}$ Pour d'autres exemples, cf. Larrory (2006).

${ }^{11}$ Cf. note 3.

${ }^{12}$ Le verbe sammeln renvoie à l'action de 'cueillir', de 'collecter' et de 'collectionner'
} 
Tout ce qu'on peut collectionner (n'est-ce pas). Depuis les timbres, les couvercles de laits concentrés accompagnant le café, les pins, les flacons de parfums jusqu'aux dés à coudre, cartes postales, boîtes d'allumettes - tout trouve collectionneur. La plupart des collectionneurs se concentrent sur des objets peu onéreux, qui prennent de la valeur avec le temps et pourraient peut-être un jour rapporter une belle somme. (trad. ALW)]

Pour P.-Y. Modicom (2016), « doch est [...] le marqueur du rappel à l'ordre » (p. 83). Par la présence de doch dans l'énoncé exclamatif en was... alles, le journaliste force l'assentiment quant au point de vue qu'il fait valoir ( 'on peut collectionner beaucoup de choses'), le rappelant à l'interlocuteur contre tout ce qui semblerait induire le point de vue contraire (y compris, ici, l'oubli). Il fait ainsi de l'énoncé exclamatif un argument fort à l'appui de l'énoncé qui précède tout en impliquant le co-énonciateur dans son énonciation.

Dans un nombre important d'énoncés, figure devant alles le négateur nicht, que l'on pourrait qualifier d'explétif, car il ne sert pas à nier le contenu propositionnel :

(9) Ich ging also mit. Was tut man nicht alles aus Liebe! Später, im Falle eines Ehekrachs, würde ich auftrumpfen können: „Sogar Kurkonzerte habe ich mir um deinetwillen angehört — und du wirfst mir vor, ich hätte dich nie geliebt..." (Martin, Einer fehlt zum Kurkonzert, p. 39)

[Je l'accompagnai donc. Que ne fait-on pas par amour ! Plus tard, en cas de scène de ménage, je pourrais alléguer : “Pour toi, j'ai même assisté à des concerts de cure thermale, et tu me reproches de ne t'avoir jamais aimée ". (trad. ALW)]

L'ajout de nicht produit l'effet d'un commentaire plus étonné et d'une sollicitation du co-énonciateur pour qu'il partage la position du locuteur. Même si nicht alles suppose un ensemble d'objets, il autorise à n'en citer qu'un seul, celui qui, précisément, déclenche l'énoncé exclamatif. Avec alles seul, le lecteur recherche dans le co-texte ou la situation de parole différents éléments d'un ensemble. Nicht alles renvoie aussi à un ensemble d'objets, mais un seul objet peut être cité (ici : entendre des concerts de cure thermale). Le reste est laissé à l'imagination du lecteur.

L'énoncé exclamatif de l'extrait (9), comme celui de l'extrait (8), implique en outre le co-énonciateur par son caractère généralisant : partant du déictique ich [je] dans le récit, le narrateur glisse vers man [on] dans le commentaire exclamatif pour revenir à ich ensuite. Or, l'opération de généralisation permet de rendre l'énoncé instanciable non seulement par le ich lui-même mais aussi par l'ensemble de référents parmi lesquels le co-énonciateur ${ }^{13}$, invité (voire

${ }^{13}$ Cf. Ali Bouacha (1993) : « l'énoncé généralisant se caractérise moins par l'absence de repérages situationnels que par sa disponibilité énonciative qui le rend à la fois itéré et itérable. Il ne renvoie pas à un discours sans sujets mais au contraire à un discours de tous les sujets. » et Daux-Combaudon (2012). 
forcé), de ce fait, à co-énoncer le commentaire exclamatif. Ce man généralisant est, avec d'autres formes généralisantes ou impersonnelles (par exemple sich lassen en (8)), extrêmement fréquent en association avec l'exclamative en was... (nicht) alles.

Dans l'énoncé exclamatif de l'extrait suivant, on trouve ainsi un uns [nous], qui implique de manière comparable à man le co-énonciateur. Mais on y trouve aussi un autre marquage fréquemment associé aux exclamatives en was... alles : le déictique de manière / intensité so [ainsi, si, aussi].

(10) In Holland haben sie jetzt Hundefleisch in Hackbällchen gefunden, in Schweden Schwein in Elch-Lasagne. Verrückte Welt aber auch. Was uns nicht alles so aufgetischt wird. Und wir futtern es. Die armen Elche. Unser Thema heute: Augen auf beim Lasagne-Kauf! (DeReKo, Braunschweiger Zeitung, 11.04.2013)

[En Hollande ils ont trouvé de la viande de chien dans des boulettes, en Suède du porc dans des lasagnes à la viande d'élan. Le monde ne tourne pas rond.

Que ne met-on pas dans nos assiettes. Et nous le bouffons. Pauvres élans. Notre sujet du jour : ouvrez les yeux quand vous achetez des lasagnes. (trad. ALW)]

So, déictique de manière, qui connaît de multiples emplois en allemand, fonctionne ici comme un "marqueur de flou » et permet de renvoyer à une expérience typique que l'énonciateur suppose partagée par le co-énonciateur et qu'il l'invite à convoquer ${ }^{14}$.

Tous ces indices vont dans le même sens : l'énoncé en was (doch) (nicht) (so) alles vise à faire partager par le co-énonciateur le commentaire exprimé par l'énoncé voire à le faire contribuer par l'imagination à la représentation que soulève cet énoncé. La configuration exclamative que nous examinons à présent est assez différente.

\subsection{L'ÉNONCÉ EN VIELLEICHT}

Dans la plupart de ses emplois, vielleicht est un modalisateur par lequel le locuteur indique que ce qu'il décrit n'est pas entièrement certain.

(11) Es wird vielleicht regnen.

Il va peut-être pleuvoir.]

Mais vielleicht connaît aussi un emploi remarquable dans l'exclamation :

(12) „Na, nu ma’ keene Komplimente!“ lachte er laut. „Dit jibt's doch ja nich, daß eena auß'n Westen sich uff'n Osten freut", schallte es durch den Flur.

„Warum nicht? Bis jetzt habe ich noch nicht viel von Oststadt kennengelernt, aber die Umgebung ist traumhaft schön. Das sind vielleicht Landschaften!“ steigerte ich möglichst glaubwürdig meine Begeisterung. (Endlich, Neuland, p. 18)

${ }^{14}$ Cf. Larrory-Wunder (2009 et 2012). 
[« Allons, allons pas de compliments, dit-il en riant bruyamment. Quand on vient de l'Ouest, on ne se réjouit pas d'aller à l'Est ». Ses paroles résonnaient dans le couloir.

«Pourquoi pas ? Jusqu'à présent, je n'ai pas encore découvert grand-chose de Oststadt, mais les alentours sont de toute beauté. Quels paysages ! [litt. ce sont peut-être des paysages]. Je redoublai d'enthousiasme en essayant de rester le plus crédible possible. (trad. ALW)]

(13) Ihr seid vielleicht Helden (oral)

[litt. : Vous êtes peut-être des héros / Quels héros ! (ironique)]

(14) Das war vielleicht ekelhaft (oral)

[litt. C'était peut-être dégoûtant. / Qu'est-ce que c'était dégoûtant !]

En synchronie au moins, on a l'emploi d'un modalisateur dans un contexte qui impose clairement une interprétation non modalisatrice de l'énoncé. En (12), il ne s'agit pas pour le locuteur de mettre en doute qu'il a affaire à des paysages mais de signifier que les paysages en question sont particulièrement beaux. L'énoncé en vielleicht sollicite un travail interprétatif, semblable à ce qui se passe avec la litote ou l'ironie, d'autant qu'il comporte souvent aussi, comme en (13), des expressions métaphoriques ou ironiques. Le locuteur produit un énoncé qui est manifestement en deçà de ce qu'il veut vraiment signifier voire qui exprime une opinion qu'il ne peut que rejeter. C'est au récepteur de comprendre, à partir du contexte, que l'intention communicative ne correspond pas à ce qui est littéralement exprimé par l'énoncé. Il est intéressant de remarquer que les énoncés en vielleicht n'apparaissent pas dans des contextes monologaux, mais dans des interactions. Deux contextes d'emplois sont particulièrement fréquents :

- produire un commentaire sur une attitude de l'interlocuteur (13)

- produire au sein d'un récit oral à la première personne un énoncé donnant accès à un état intérieur du locuteur et en faisant partager l'émotion $(14)^{15}$.

Dans ces contextes, vielleicht apparaît comme un adoucisseur, permettant de ménager les partenaires de la communication, en formulant un reproche sous une forme détournée, mieux acceptable (13), ou en exprimant émotions ou sensations avec une certaine retenue (14). Comme c'est le cas des particules énonciatives (telles que doch vu plus haut), vielleicht est, dans cet emploi, obligatoirement en position insérée, médiane, et l'énoncé intègre en son sein, en la désamorçant en quelque sorte, la possibilité d'une position contraire à celle que le locuteur fait valoir.

${ }^{15}$ Cf. aussi Larrory (2003). 
L'interprétation exclamative est guidée par l'accent, un accent inhabituel, initial, frappant l'élément déictique ${ }^{16}$, qui démarque l'énoncé du contexte et constitue un élément supplémentaire de sollicitation de l'attention de l'allocuté.

À la différence des énoncés en was... alles, les énoncés en vielleicht ne servent pas, nous semble-t-il, à susciter une représentation commune partagée. Dans une situation d'interlocution, ils permettent plutôt, pour le locuteur, de produire un jugement qui lui tient à cœur, tout en évitant une expression directe de ce jugement, pour tenir compte de la position de l'allocuté : le commentaire produit peut en effet porter sur l'allocuté lui-même et représenter par là une 《 menace ${ }^{17}$ qu'il est préférable, par avance, d'atténuer ; il peut, au contraire, porter sur des états internes du locuteur lui-même, par nature inaccessibles à autrui et qu'il s'agit pour le locuteur de proposer prudemment à l'empathie de l'allocuté.

Le dispositif énonciatif que déploient certaines formes exclamatives en wenn [si] et que nous allons examiner à présent se rapproche de celui des énoncés en vielleicht, mais avec un effet différent.

\subsection{ENONCÉS EN WENN}

Comparables aux énoncés exclamatifs en si correspondants en français, les énoncés en wenn sont constitués de « subordonnées indépendantes », structurellement en attente de complétude, mais que, dans un contexte exclamatif, rien ne vient achever. Ils sont bâtis sur une structure attributive, dans laquelle le sujet est un pronom en $d$ - (à valeur démonstrative) et l'attribut un groupe nominal qualifiant (dans notre corpus du moins) et qui se présente le plus souvent (mais pas toujours, cf. 17) à la forme négative :

(15) „,...) Was wollen Sie überhaupt von Herrn Gollmer?“

„Ich möchte — ich will — ich habe etwas Geschäftliches mit ihm zu besprechen!“

„Und da kommen Sie hierher?! Hier redet Vater nie über Geschäfte! Das ist ganz nutzlos, er hört Sie gar nicht erst an, er schmeißt Sie auf der Stelle raus!“ Sie sah ihn strafend an.

Ihr Vater... Der große Automobilkaufmann Gollmer war ihr Vater! Wenn das kein Wink des Himmels war! (Fallada, Ein Mann will nach oben, p. 230-231)

[- Et d'ailleurs, que lui voulez-vous, à M. Gollmer ?

- Je voudrais - je veux - je dois parler affaires avec lui !

${ }^{16}$ P.-Y. Modicom (2016) interprète cet accent, en lien avec vielleicht, comme un accent paradigmatisant, qui singularise le sujet au sein d'une classe.

${ }^{17}$ Cf. Brown / Levinson (1987: 65sq.) : face-threatening act. 
- Et vous venez ici ?! Mon père ne parle jamais de ses affaires ici. C'est inutile, il ne vous écoutera pas, il vous mettra dehors sur-le-champ !

Elle le regarda d'un air sévère.

Son père... Le grand marchand de voitures Gollmer était son père ! Si ce n'était pas un signe du ciel ! (trad. ALW)]

(16) Liebe Schweiz, 150 Jahre hält die Bundesverfassung schon. Wenn das keinen Glückwunsch wert ist! (DeReKo, Braunschweiger Zeitung, 21.01.2013)

[Chère Suisse, déjà 150 ans de cette constitution fédérale. Si cela ne mérite pas des félicitations ! (trad. ALW)]

(17) „,...) Aber wie du mit Rieke umgehst, das ist eine Hundsgemeinheit, das sage ich dir! Ich habe dich beizeiten gewarnt, aber da warst du ganz groß, Rieke war deine Heimat, Rieke liebtest du! Wenn das deine Liebe ist — ! So möchte ich nicht zu meinem Dienstmädchen sein, so rede ich nicht zu einer Nutte, wie du mit Rieke sprichst! Ein eiskalter Kerl bist du! (...)“ (Fallada, Ein Mann will nach oben, p. 311)

[,Mais la manière dont tu traites Rieke, c'est une saloperie, je te le dis ! Je t'ai déjà averti quand c'était le moment, mais tu étais grand, Rieke, c'était ton pays, tu aimais Rieke ! Si c'est ça, ton amour ! Je ne voudrais pas me comporter ainsi avec ma domestique, à une pute je ne parle pas comme tu parles à Rieke ! Tu es sans cœur ! »]

Comme l'énoncé en vielleicht, l'énoncé en wenn range le contenu propositionnel dans l'ordre du 'possible', mais il suggère systématiquement la validité du contenu contraire : 'c'est un signe du ciel' / 'cela mérite des félicitations' / 'ce n'est pas de l'amour'. A gauche (et parfois à droite) du commentaire exclamatif en wenn, on trouve une mention du fait qui le déclenche ; en (17), cette mention prend la forme d'un discours rapporté des propos de l'interlocuteur: Rieke war deine Heimat, Rieke liebtest du). L'énoncé en wenn réagit ainsi à ces propos en manifestant que le locuteur ne partage pas le point de vue en question. En (15) et (16), on peut considérer que cette confrontation de deux positions reste imaginaire : l'énonciateur feint simplement de reprendre une assertion antérieure fictive pour la rejeter et forcer l'adhésion. En fait, cette assertion fictive n'est même pas toujours énonçable (énoncer das war kein Wink des Himmels, par exemple, est étrange) ; elle n'existe que comme un « envers » imaginaire. Le dispositif énonciatif met donc ici en scène, par la forme en wenn appliquée à un contenu propositionnel attributif, un énonciateur imaginaire auquel peut être identifié le co-énonciateur, dont la position se trouve invalidée à la fois par la situation que commente l'énoncé exclamatif en wenn et par la configuration même de l'énoncé. 


\section{DES AFFINITÉS DE L'EXCLAMATION AVEC L'INJONCTION}

Les phénomènes de sollicitation ou de ménagement de l'allocuté et de renvoi à une instance énonciative imaginaire se retrouvent de manière très intéressante dans des énoncés injonctifs qui ont des affinités avec les énoncés exclamatifs (18 à 22). Gaubert (2000) relève ainsi l'emploi du verbe sich vorstellen ('s'imaginer') dans le co-texte gauche ou droit d'un énoncé exclamatif, à la forme impérative (Stell dir vor 'Imagine-toi' / Stellen Sie sich vor 'Imaginez-vous') ou dans un énoncé « semi-injonctif » avec un verbe de modalité (Das muss man sich mal vorstellen 'Il faut s'imaginer'). En (18) et (19) sont employés respectivement avant puis après l'exclamative un énoncé à l'impératif comportant un verbe de perception et un énoncé à l'impératif comportant un verbe de dire :

(18) Du lebst noch im selben Haus, aber in einem höheren Stock.

Auf einer anderen Ebene, in einer anderen Wohnung. Merkst du es denn nicht, daß dein Zimmer kleiner geworden ist? Auch die Möbel, der Schrank, der Spiegel — Du kannst dich noch sehen im Spiegel, er ist immer noch groß genug — gewiß, gewiß! Du bist auch nur ein Mensch, der möchte, daß seine Krawatte richtig sitzt. Doch sieh mal zum Fenster hinaus!

Wie entfernt ist alles geworden! Wie winzig sind plötzlich die großen Gebieter und wie arm die reichen Plebejer! Wie lächerlich!

Wie verwaschen die Fahnen!

Kannst du die Transparente noch lesen?

Nein.

Hörst du noch das Radio?

Kaum. (Horvath, Jugend ohne Gott, p. 113)

[Tu habites toujours la même maison, mais à un étage supérieur.

À un autre niveau, dans une autre demeure. Ne vois-tu pas que ta chambre a rapetissé ? Les meubles aussi, l'armoire, le miroir. - Tu te vois toujours dans le miroir, il est encore assez grand - certes, certes ! Tu n'es qu'un homme, après tout, qui voudrait que sa cravate soit bien droite. Mais regarde par la fenêtre !

Comme tout te paraît lointain à présent ! Comme ils sont insignifiants les gros propriétaires, comme ils sont pauvres les riches plébéiens ! Comme ils sont risibles !

Comme ils sont délavés les drapeaux !

Peux-tu encore lire les banderoles?

Non.

Entends-tu encore la radio ?

À peine. (trad. Lambrechts, p. 152-153)] 
(19) „(...) Zum Ablecken war sie. Zum Austrinken war sie. Ach Gott, ach Gott war sie schön. So ein Frauenzimmer! Nein sag mir nur wie kann es so ein Frauenzimmer geben?“ (Kafka, Der Verschollene, p. 234)

[A croquer qu'elle était. A siroter ! Dieu de Dieu, qu'elle était belle ! Quelle femme ! Non mais, dis-moi, comment peut-il exister une femme pareille ? (trad. Lortholary, p. 246-247)]

Les deux exemples cités montrent au moins deux fonctions de l'injonction - souvent routinière — dans ce contexte : précédant l'exclamation, l'injonction sert à attirer l'attention sur des objets du monde, ceux-là mêmes qui feront l'objet d'une réaction exclamative (sieh mal zum Fenster hinaus, stell dir vor...) ; succédant à l'exclamation, l'injonction cherche à impliquer l'allocuté, à lui faire partager la position du locuteur (Nein sag mir nur wie kann es so ein Frauenzimmer geben?).

Mais on trouve aussi des formes qui peuvent être, selon nous, interprétées comme des injonctions indirectes :

(20) Der Bimbo hat sein Goldkettchen jetzt schon zum dritten mal von seinem roten Fitness-Hals genommen und mit dem Fingernagel den Dreck zwischen den einzelnen Gliedern herausgestochert. „Ein Wahnsinn, wie schnell die kreuzgeschweißten Glieder verdrecken!“" hat der Bimbo so zornig geschrien, als müßte er einem Zivildiener das Autowaschen beibringen.

Aber es ist nur sein Goldkettchen gewesen, das er zusammengeschnauzt hat. Die Zivildiener hat man ihnen ja schon vor zwei Jahren weggenommen. Seither ist es bergab gegangen mit dem Vorsprung gegenüber dem Rettungsbund. Aber der Bimbo ist trotzdem froh gewesen, daß er keinen von diesen Schlappschwänzen mehr gesehen hat.

„Ich frage mich, wo der ganze Dreck eigentlich herkommt!“ hat der Bimbo sein Goldkettchen zusammengeschnauzt. „Wenn du dir vorstellst, daß dieser ganze Dreck in der Luft ist! Und wir schnaufen diesen Dreck die ganze Zeit ein!“ (Haas, Komm süßer Tod, p. 19)

Pour la troisième fois, Bimbo avait ôté de son cou rouge body-buildé la petite chaîne en or et en avait nettoyé les maillons avec ses ongles. «C'est quoi, toute cette crasse ? » a aboyé Bimbo, comme lorsqu'il inspectait les voitures lavées par les objecteurs de conscience.

Mais il en avait seulement après sa chaîne en or. Ils n'avaient plus d'objecteurs de conscience depuis deux ans. Ensuite, l'avance sur l'ABUSA avait fondu comme neige au soleil. Mais Bimbo était tout de même bien content d'être débarrassé de ces lavettes.

Bimbo s'en est pris à sa chaîne en or : « J'aimerais bien savoir d'où elle vient, cette crasse ! Et dire que c'est la pollution ! [litt : Si tu imagines que toute cette crasse est dans l'air] Et qu'on s'en met plein les poumons toute la journée ! » (trad. Reygnier, p. 25) 
Cette forme en wenn est paraphrasable par une forme à l'impératif :

Stell dir vor, dass dieser ganze Dreck in der Luft ist. [Imagine-toi que toute cette crasse est dans l'air]

Elle rappelle des formes injonctives indirectes en wenn, dont l'emploi sert une visée de politesse :

Wenn Sie mir folgen wollen. [Si vous voulez bien me suivre]

La tournure figée dass ich nicht lache peut également s'interpréter comme une injonctive indirecte comme dans les contextes (21) et (22) issus de forums sur Internet :

(21) [a] Altenpflege - ein sozialer Beruf?

[b] Dass ich nicht lache.

(http://www.job-hilfe.de/beruf/altenpfleger/649-meine-bilanz-altenpflege-ein-sozialer-beruf-dass-ich-nicht-lache, 23.5.2016)

[Aide-soignant auprès des personnes âgées. Un métier social. Laissez-moi rire.]

(22) $02.03 .201514: 48$

merkasan schreibt

[a] android zu dem preis? [b] dass ich nicht lache.

(http://www.teltarif.de/forum/s58350/android-zu-dem-preis-dass-ich-nichtlache/10.html, 23.5.2016)

[merkasan écrit

Android à ce prix. Laissez-moi rire. (litt. que je ne rie pas)]

Dans ce type d'exemples on a systématiquement un premier segment prédicatif [a] constitué d'éléments repris du contexte et présenté sur un mode interrogatif, puis la forme d'énoncé en dass [b] qui vient rejeter l'association prédicative proposée par ce segment. Si cette tournure en dass est figée, on peut l'interpréter, par analogie avec d'autres formes en dass dans la langue ${ }^{18}$, comme injonctive et opérant par un procédé de renversement : c'est à un co-énonciateur imaginaire qu'est attribuée l'injonction qui concerne l'énonciateur (ich), tandis que l'attitude de celui-ci / le contexte d'énonciation donne à entendre que la requête imaginaire est intenable. Une reformulation possible de l'énoncé en dass est d'ailleurs la suivante : Da muss ich aber lachen [Là, je suis obligé de rire].

${ }^{18} \mathrm{Il}$ est possible en allemand de formuler des injonctions expressives en dass : Dass $d u$ mir ja pünktlich bist! [litt. Que tu sois à l'heure!]. 


\section{CONCLUSION}

Un locuteur qui s'exclame commente un état de fait qui l'affecte. Mais les observations que nous avons faites obligent à considérer que ce commentaire ne concerne pas le seul locuteur / énonciateur ; il intègre manifestement ce que l'on pourrait appeler une "image » de la relation de celui-ci à un autre que luimême, que ce soit l'interlocuteur/lecteur/co-énonciateur invité à partager un point de vue, l'allocuté en chair et en os qu'il faut ménager, le co-énonciateur construit comme contre-position fictive à partir de laquelle le locuteur/énonciateur impose, de manière forte, son point de vue. Certes, la plupart des énoncés exclamatifs (à l'exception de l'énoncé en vielleicht) ne nécessitent ni interlocution ni présence physique d'un allocuté et s'accommodent bien du monologue ; mais, pour les cas que nous avons exposés, ils construisent, dans leurs marquages mêmes, une position imaginaire pour l'autre, qui est envisagé tantôt comme récepteur imaginé du message, invité éventuellement à le co-énoncer, tantôt comme émetteur fictif d'une contre-proposition. Ainsi le point de vue qu'expriment ces énoncés ne se donne-t-il pas simplement comme subjectif mais comme intersubjectivement partageable voire comme produit dans un rapport intersubjectif et c'est sans doute ce qui confère à l'énoncé exclamatif sa force expressive si originale ${ }^{19}$.

${ }^{19}$ Nous rejoignons finalement ici le diagnostic de Kerfelec (2009 : p. 246sq.), que confirme le jeu des marquages que nous avons analysé : « les exclamatives ne relèvent pas du monologue ou du soliloque mais sont bien de nature dialogale parce qu'elles reposent sur un rapport (de force) entre L1 et L2 (...) Loin d'émaner d'une conscience isolée qui ne peut sortir d'elle-même, elles marquent la rencontre de deux consciences face à une évidence. » (p. 252-253). 


\section{RÉFÉRENCES BIBLIOGRAPHIQUES}

Ali Bouacha, A. (1993), «Enonciation, argumentation et discours: le cas de la généralisation », Semen [En ligne], 8 | 1993, mis en ligne le 06 juillet 2007, consulté le 14 janvier 2017. URL : http://semen.revues.org/3985.

Altmann, H. (1993), « Satzmodus », in J. Jacobs / A. v. Stechow / W. Sternefeld / T. Vennemann (éd.), Syntax: Ein internationales Handbuch der zeitgenössischen Forschung, Berlin: de Gruyter, p. 1006-1029.

Brown, P. / Levinson, S. C. (1987), Politeness: Some universals in language usage, Cambridge : CUP.

Bücker, J. (2012), Sprachhandeln und Sprachwissen: Grammatische Konstruktionen im Spannungsfeld von Interaktion und Kognition, Berlin : de Gruyter.

Culioli, A. (2002), Variations sur la linguistique, Paris : Klincksieck.

Daux-Combaudon, A.-L. (2012), « Verallgemeinernde Äußerungen zwischen Verständnissicherung und Unterhaltsamkeit », in A. Larrory-Wunder (éd.), Intersubjektivität und Sprache. Zur An- und Abgleichung von Sprecher- und Hörervorstellungen in Gesprächen und Texten, Tübingen : Stauffenburg, p. 67-89.

d'Avis, F.-J. (2001), Über >w-Exklamativsätze< im Deutschen (= Linguistische Arbeiten, 429), Tübingen : Niemeyer.

d'Avis, F.-J. (2013), « Exklamativsatz », in J. Meibauer / M. Steinbach / H. Altmann (éd.), Satztypen des Deutschen, Berlin : de Gruyter, p. 171-201.

Ducrot, O. (1984), Le dire et le dit, Paris : Minuit.

Fries, N. (1988), « Ist Pragmatik schwer ! Über sog. „Exklamativsätze“ im Deutschen », Sprache und Pragmatik, 3, p. 1-18.

Gaubert, C. (2001), Les énoncés exclamatifs en allemand moderne, Thèse de doctorat, Université de Rennes II.

Guillaume, B. / Baumer, E. (2014), « Expression de l'exclamation en anglais au moyen de marqueurs atypiques », CORELA 12-2.

Kerfelec, V. (2009), L'exclamation en français et en anglais. Formes, sens, effets, Aixen-Provence : Publications de l'Université de Provence.

Larrory, A. (2002), Was du nicht sagst ! Les énoncés exclamatifs en allemand moderne, Thèse de doctorat, Université de Reims.

Larrory, A. (2003), «Vielleicht exclamatif », Nouveaux cahiers d'allemand 2003/3, p. 325-337.

Larrory, A. (2004), « Mehr oder weniger exklamativ? Wie kann man Exklamation definieren? », in M. Krause / N. Ruge (éd.), Das war echt spitze! Zur Exklamation im heutigen Deutsch (= Eurogermanistik, 21), Tübingen : Stauffenburg, p. 1-14. 
Larrory, A. (2006), « Partition et quantification dans les énoncés exclamatifs en allemand », in G. Kleiber / C. Schnedecker / A. Theissen (éd.), La relation partie-tout, Louvain / Paris / Dudley : Peeters, p. 303-318.

Larrory-Wunder, A. (2009), «L'absence d'ancrage dans certains emplois du déictique so en allemand », in A. Grutschus / P. Krilles (éd.), Figuren der Absenz - Figures de l'absence, Berlin : Frank \& Timme, p. 235-248.

Larrory-Wunder, A. (2012), « So als Angleichungsmarker », in A. Larrory-Wunder (éd.), Intersubjektivität und Sprache. Zur An- und Abgleichung von Sprecher- und Hörervorstellungen in Gesprächen und Texten, Tübingen : Stauffenburg, p. 1-24.

Martin, R. (1987), Langage et croyance. Les " univers de croyance » dans la théorie sémantique, Bruxelles : Pierre Mardaga.

Métrich, R. / Faucher, E. / Courdier, G. (1999), Les invariables difficiles, tome 2, Nancy : Bibliothèque des Nouveaux Cahiers d'Allemand.

Modicom, P.-Y. (2016), L'énoncé et son double. Recherches sur le marquage de l'altérité énonciative en allemand. Thèse de doctorat, Université Paris-Sorbonne.

Näf, A. (1987), « Gibt es Exklamativsätze ?», in J. Meibauer (éd.), Satzmodus zwischen Grammatik und Pragmatik (= Linguistische Arbeiten, 180), Tübingen : Niemeyer, p. 140-160.

Rabatel, A. (1998), La construction textuelle du point de vue, Lausanne : Delachaux \& Niestlé.

Reis, M. (1992), « The Category of Invariant alles in Wh-Clauses: On Syntactic Quantifiers vs. Quantifying Particles in German », in R. Tracy (éd.), Who Climbs the Grammar-Tree (=Linguistische Arbeiten, 281), Tübingen : Niemeyer, p. 465-492.

Rosengren, I. (1992), «Zur Grammatik und Pragmatik der Exklamation », in I. Rosengren (éd.), Satz und Illokution (= Linguistische Arbeiten, 278, Tübingen : Niemeyer, p. 263-306.

Valentin, P. (1989), «A propos des énoncés exclamatifs allemands », in E. Faucher / F. Hartweg / J. Janitza (éd.), Sens et Être. Mélanges en l'honneur de J.-M. Zemb, Nancy : Presses universitaires, p. 243-248.

Watine, M.-A. (2015), « Les âges de la réduplication », Semen [En ligne], 38 | 2015, mis en ligne le 24 avril 2015, consulté le 13 janvier 2017. URL : http://semen.revues. org/10309 


\section{SOURCES CITÉES}

Corpus :

DeReKo : Deutsches Referenzkorpus, Mannheim, Institut für Deutsche Sprache.

Romans :

Endlich, L. (1999), NeuLand. Ganz einfache Geschichten, Berlin, Transit.

Fallada, H. (1994), Ein Mann will nach oben, Hamburg, Rowohlt.

Fallada, H. (1998/1932), Kleiner Mann, was nun?, Hamburg, Rowohlt.

Haas, W. (1998), Komm, süßer Tod, Hamburg, Rowohlt. [trad. M. Reygnier, Vienne la mort, Paris, Rivages, 2002]

Härtling, P. (1988), Felix Guttmann, Darmstadt, Luchterhand. [trad. C. Porcell, Felix Guttmann, Paris, Seuil, 1989]

Hilbig, H. (2000), Das Provisorium, Frankfurt-am-Main, Fischer. [trad. B. Vergne-Cain et G. Rudent, Provisoire, Paris, Métailié, 2004]

Horvath, Ö. (1983/1938), Jugend ohne Gott, Frankfurt-am-Main, Suhrkamp. [trad. R. Lambrechts, Jeunesse sans dieu, Paris, Christian Bourgois, 2002]

Kafka, F. (1994/1927), Der Verschollene, Frankfurt-am-Main, Fischer Taschenbuch. [trad. B. Lortholary, Amerika ou le Disparu, Paris, Flammarion, 1988]

Martin, H. (1991), Der Rest ist sterben, Hamburg, Rowohlt.

Martin, H. (1967), Einer fehlt zum Kurkonzert, Hamburg, Rowohlt.

Film :

Fassbinder, R. W. (1974), Angst essen Seele auf. 